\title{
Two New Integrable Couplings of Soliton Hierarchies with Self-consistent Sources
}

\author{
Sixing Tao and Hui Shi
}

\begin{abstract}
A new kind of integrable couplings of soliton hierarchies with self-consistent sources associated with $\widetilde{s l}(4)$ was presented. Based upon this method, two new integrable couplings associated with Li soliton hierarchy and BKK soliton hierarchy with self-consistent sources were obtained by making use of loop algebra $\widetilde{s l}(4)$ respectively. Meanwhile, their Hamiltonian structures of the integrable couplings of $\mathrm{Li}$ and BKK soliton hierarchies were obtained by making use of variational identity. The method in this study can be applied to other soliton hierarchies with self-consistent sources.
\end{abstract}

Index Terms-Li soliton hierarchy, self-consistent sources, integrable couplings, BKK soliton hierarchy

\section{INTRODUCTION}

Soliton equations with self-consistent sources [1]-[4] has been receiving growing attention in recent years. Physically, the sources may result in solitary waves with a non-constant velocity and therefore lead to a variety of dynamics of physical models. For applications, these kinds of systems are usually used to describe interactions between different solitary waves and are relevant to some problems of hydrodynamics, solid state physics, plasma physics, etc.

Recently, the integrable couplings with self-consistent sources have been receiving growing attention. Yu [5]-[7] has derived some integrable couplings of soliton hierarchy with self-consistent sources such as the Dirac soliton hierarchy with self-consistent sources, the Yang soliton hierarchy with self-consistent sources and the $\mathrm{C}-\mathrm{KdV}$ soliton hierarchy with self-consistent sources by making use of the loop algebra $\tilde{s l}(4)$, but there are some errors in [5]-[7] . In this paper, we will correct these errors and construct a new integrable couplings of soliton hierarchy with self-consistent sources by making use of loop algebra $\widetilde{s l}(4)$.

This paper is organized as follows. In section II, we will lead to a kind of integrable couplings of soliton hierarchies with self-consistent sources by making use of $\widetilde{s l}(4)$. In section III, integrable couplings of the Li soliton hierarchy with self-consistent sources is derived by making use of loop algebra $\widetilde{s l}(4)$. In section IV, integrable couplings of the

Manuscript received March 29, 2011; revised June 2, 2011.

This work was supported by the Youth Research Foundation of Shangqiu Normal University under Grant

No. 2011QN12, the Natural Science Foundation of China under Grant No. 61072147 .

Sixing Tao is with the Department of Mathematics, Shangqiu Normal University, Shangqiu, 476000, China (tel: 86-13781680641; email: taosixing@163.com).

Hui Shi is with the Department of Physics and Information Engineering, Shangqiu Normal University, Shangqiu, 476000, China (tel: 86-13523157699, email: shihui529@126.com).
Broer-Kaup-Kupershmidt soliton hierarchy with self-consistent sources is derived by making use of loop algebra $\widetilde{s l}(4)$. Finally, some conclusions are given.

\section{A New InTEgrable COUPLings OF SOLITON} HIERARCHY WITH SELF-C ONSISTENT SOURCES ASSOCIATED WITH $\tilde{s l}(4)$

In the following, we consider a set of matrix Lie algebra $s l(4)[5$

$$
\begin{aligned}
g_{1} & =\left(\begin{array}{cccc}
1 & 0 & 0 & 0 \\
0 & -1 & 0 & 0 \\
0 & 0 & 1 & 0 \\
0 & 0 & 0 & -1
\end{array}\right), g_{2}=\left(\begin{array}{llll}
0 & 1 & 0 & 0 \\
1 & 0 & 0 & 0 \\
0 & 0 & 0 & 1 \\
0 & 0 & 1 & 0
\end{array}\right), \\
g_{3} & =\left(\begin{array}{cccc}
0 & 0 & 0 \\
-1 & 0 & 0 & 0 \\
0 & 0 & 1 & 1 \\
0 & 0 & -1 & 0
\end{array}\right), g_{4}=\left(\begin{array}{cccc}
0 & 0 & 1 & 0 \\
0 & 0 & 0 & -1 \\
0 & 0 & 0 & 0 \\
0 & 0 & 0 & 0
\end{array}\right), \\
g_{5} & =\left(\begin{array}{llll}
0 & 0 & 0 & 1 \\
0 & 0 & 1 & 0 \\
0 & 0 & 0 & 0 \\
0 & 0 & 0 & 0
\end{array}\right), g_{6}=\left(\begin{array}{cccc}
0 & 0 & 0 & 1 \\
0 & 0 & -1 & 0 \\
0 & 0 & 0 & 0 \\
0 & 0 & 0 & 0
\end{array}\right)
\end{aligned}
$$

It is easy to verify that

$$
\begin{aligned}
& \operatorname{sl}(4)=\operatorname{span}\left\{\mathrm{g}_{1}, \mathrm{~g}_{2}, \mathrm{~g}_{3}, \mathrm{~g}_{4}, \mathrm{~g}_{5}, \mathrm{~g}_{6}\right\} \\
& \operatorname{sl}(4)_{1}=\operatorname{span}\left\{\mathrm{g}_{1}, \mathrm{~g}_{2}, \mathrm{~g}_{3}\right\}, \\
& \mathrm{sl}(4)_{2}=\operatorname{span}\left\{\mathrm{g}_{4}, \mathrm{~g}_{5}, \mathrm{~g}_{6}\right\}
\end{aligned}
$$

Construct three Lie algebras, and satisfy

$$
s l(4)=\operatorname{sl}(4)_{1} \oplus \operatorname{sl}(4)_{2} \text {, }
$$

$$
\left[s l(4)_{1}, s l(4)_{2}\right]=\operatorname{sl}(4)_{1} s l(4)_{2}-\operatorname{sl}(4)_{2} s l(4)_{1} \subseteq \operatorname{sl}(4)_{2} .
$$

The corresponding loop Lie algebra are defined as follows:

$$
\begin{aligned}
\widetilde{s l}(4) & =\left\{A \mid A \in R\left[\lambda, \lambda^{-1}\right] \otimes s l(4)\right\}, \\
\widetilde{s l}(4)_{1} & =\left\{A \mid A \in R\left[\lambda, \lambda^{-1}\right] \otimes s l(4)_{1}\right\}, \\
\widetilde{s l}(4)_{2} & =\left\{A \mid A \in R\left[\lambda, \lambda^{-1}\right] \otimes s l(4)_{2}\right\} .
\end{aligned}
$$

Consider the auxiliary linear problem

$$
\begin{aligned}
& \left(\begin{array}{l}
\phi_{1} \\
\phi_{2} \\
\phi_{3} \\
\phi_{4}
\end{array}\right)_{x}=U(u, \lambda)\left(\begin{array}{l}
\phi_{1} \\
\phi_{2} \\
\phi_{3} \\
\phi_{4}
\end{array}\right), \\
& \left(\begin{array}{l}
\phi_{1} \\
\phi_{2} \\
\phi_{3} \\
\phi_{4}
\end{array}\right)_{t_{n}}=V^{(n)}(u, \lambda)\left(\begin{array}{l}
\phi_{1} \\
\phi_{2} \\
\phi_{3} \\
\phi_{4}
\end{array}\right),
\end{aligned}
$$

where

$$
U(u, \lambda)=g_{1}(\lambda)+\sum_{i=1}^{6} u_{i} g_{i}(\lambda), u=\left(u_{1}, \ldots, u_{6}\right)^{\mathrm{T}},
$$$$
u_{i}=u_{i}(x, t)(i=1,2, \ldots 6), \phi_{j}=\phi_{j}(x, t)(\mathrm{j}=1,2,3,4)
$$

are field variables defining on $x \in R, t \in R, g_{\mathrm{i}}(\lambda) \in \widetilde{s l}(4)$, $\widetilde{s l}(4)$ denotes a infinite Lie algebra over $R$. When $\frac{\partial \lambda}{\partial t}=0$, the compatibility conditions of (3) gives rise to the well-known zero curvature equation

$$
U_{t}-V_{x}^{(n)}+\left[U, V^{(n)}\right]=0, n=1,2, \cdots,
$$

The general scheme of searching for the consistent $V^{(n)}$ and generating a hierarchy of soliton equations was proposed as follows[8]. First, we solve the stationary zero curvature equation

$$
V_{x}=U V-V U,
$$




$$
\begin{gathered}
V=\sum_{m=0}^{\infty} V_{m}(\mathrm{u}) \lambda^{-m}= \\
\sum_{m=0}^{\infty}\left(\begin{array}{cccc}
a_{m} & b_{m}+c_{m} & d_{m} & e_{m}+f_{m} \\
b_{m}-c_{m} & -a_{m} & e_{m}-f_{m} & -d_{m} \\
0 & 0 & a_{m} & b_{m}+c_{m} \\
0 & 0 & b_{m}-c_{m} & -a_{m}
\end{array}\right) \lambda^{-\mathrm{m}} .
\end{gathered}
$$

Then we search for $\Delta_{n} \in \widetilde{s l}(4)$ such that $V^{(n)}$ can be constructed by

$$
\begin{gathered}
V^{(n)}=\sum_{m=0}^{n} V_{m}(\mathrm{u}) \lambda^{n-m_{+}}+\Delta_{n}(u, \lambda) \\
\Delta_{n}(u, \lambda)=\Delta_{n 1} g_{1}+\Delta_{n 2} g_{2}+\Delta_{n 3} g_{3}+\Delta_{n 4} g_{4}+\Delta_{n 5} g_{5}+\Delta_{n 6} g_{6}(6)
\end{gathered}
$$

where $\Delta_{n i}$ are linear functions of $a_{n}, b_{n}, c_{n}, d_{n}, e_{\mathrm{n}}$ and $f_{\mathrm{n}}$.

Under certain conditions, there is a constant $\gamma$ such that the so-called variational identity holds[9].

$$
\frac{\delta}{\delta u_{i}} \operatorname{Tr}\left(V \frac{\partial U}{\partial \lambda}\right)=\left(\lambda^{-\gamma}\left(\frac{\partial}{\partial \lambda}\right) \lambda^{\gamma}\right) \operatorname{Tr}\left(V \frac{\partial U}{\partial u_{i}}\right),
$$

where $\operatorname{Tr}$ denotes the trace of a matrix. Define a scalar $H=H(u, \lambda)$ by the equation

$$
\begin{gathered}
\left(\lambda^{-\gamma}\left(\frac{\partial}{\partial \lambda}\right) \lambda^{\gamma}\right) H=\operatorname{Tr}\left(V \frac{\partial U}{\partial \lambda}\right), \\
H=\sum_{m=0}^{\infty} H_{m}(u) \lambda^{-m} .
\end{gathered}
$$

From the variational identity (7), we obtain that

$$
\sum_{m=0}^{\infty} \frac{\delta H_{m}}{\delta u_{i}} \lambda^{-m}=\operatorname{Tr}\left(V \frac{\partial U}{\partial u_{i}}\right)=\sum_{m=0}^{\infty} \operatorname{Tr}\left(V_{m} g_{i}\right) \lambda^{-m} .
$$

The sets $\left\{H_{\mathrm{m}}\right\}$ proves the conserved densities of (4). In [8], Ma proposed the generalized $\mathrm{Tu}$ scheme to find a Hamiltonian function, a recursion operator $L$ and symplectic operator $J$ of the hierarchy based on stationary zero stationary equation (5). The Hamiltonian form with $H_{\mathrm{n}}$ can be written as

$$
\begin{gathered}
u_{t_{n}}=J \frac{\delta H_{n}}{\delta u}, n=1,2, \ldots \\
\frac{\delta H_{n}}{\delta u}=L \frac{\delta H_{n-1}}{\delta u}=\ldots=L^{n} \frac{\delta H_{0}}{\delta u}, n=1,2, \ldots, \\
\text { where } \frac{\delta}{\delta u}=\left(\frac{\delta}{\delta u_{1}}, \ldots, \frac{\delta}{\delta u_{6}}\right)^{T} .
\end{gathered}
$$

We consider the auxiliary problem of (3). For $N$ distinct $\lambda_{\mathrm{j}}, j=1, \ldots, N$, the following systems result from (3) as follows

$$
\begin{gathered}
\left(\begin{array}{l}
\phi_{1 j} \\
\phi_{2 j} \\
\phi_{3 j} \\
\phi_{4 j}
\end{array}\right)_{x}=\left(g_{1}\left(\lambda_{\mathrm{j}}\right)+\sum_{\mathrm{i}=1}^{6} u_{i} g_{i}\left(\lambda_{\mathrm{j}}\right)\right)\left(\begin{array}{l}
\phi_{1 j} \\
\phi_{2 j} \\
\phi_{3 j} \\
\phi_{4 j}
\end{array}\right), \\
\left(\begin{array}{c}
\phi_{1 j} \\
\phi_{2 j} \\
\phi_{3 j} \\
\phi_{4 j}
\end{array}\right)_{t_{n}}=\left(\sum_{m=0}^{\mathrm{n}} V_{m}(\mathrm{u}) \lambda_{\mathrm{j}}^{n-m}+\Delta_{n}\left(u, \lambda_{\mathrm{j}}\right)\right)\left(\begin{array}{c}
\phi_{1 j} \\
\phi_{2 j} \\
\phi_{3 j} \\
\phi_{4 j}
\end{array}\right)
\end{gathered}
$$

The following equation is presented in Refs.[10, 11].

$$
\frac{\delta H_{k}}{\delta u}+\sum_{j=1}^{N} \alpha_{j} \frac{\delta \lambda_{j}}{\delta u}=0,
$$

where $\alpha_{\mathrm{j}}$ are constants, $\frac{\delta H_{k}}{\delta u}$ determines a finite dimensional invariant sets for the flow (10).

For (12a), it is known (up to a constant factor) that

$$
\frac{\delta \lambda_{j}}{\delta u_{i}}=\frac{1}{2} \operatorname{Tr}\left(\Psi_{j} \frac{\partial U\left(u, \lambda_{j}\right)}{\partial u_{i}}\right)=\frac{1}{2}\left(\Psi_{j} g_{i}\left(\lambda_{j}\right)\right)
$$

where $i=1,2,3 . j=1, \ldots, N$ and

$$
\Psi_{j}=\left(\begin{array}{cccc}
\phi_{1 \mathrm{j}} \phi_{2 \mathrm{j}} & -\phi_{1 \mathrm{j}}^{2} & \phi_{3 \mathrm{j}} \phi_{4 \mathrm{j}} & -\phi_{3 \mathrm{j}}^{2} \\
\phi_{2 \mathrm{j}}^{2} & -\phi_{1 \mathrm{j}} \phi_{2 \mathrm{j}} & \phi_{4 \mathrm{j}}^{2} & -\phi_{3 \mathrm{j}} \phi_{4 \mathrm{j}} \\
0 & 0 & \phi_{1 \mathrm{j}} \phi_{2 \mathrm{j}} & -\phi_{1 \mathrm{j}}^{2} \\
0 & 0 & \phi_{2 \mathrm{j}}^{2} & -\phi_{1 \mathrm{j}} \phi_{2 \mathrm{j}}
\end{array}\right) \text {. }
$$

For $\mathrm{u}_{4}, \mathrm{u}_{5}, \mathrm{u}_{6}$, if we set $g_{i}\left(\lambda_{\mathrm{j}}\right)=\left(\begin{array}{cc}0 & A_{i j} \\ 0 & 0\end{array}\right), i=4,5,6$. $\Psi_{j 12}=$ $\left(\begin{array}{cc}\phi_{3 j} \phi_{4 j} & -\phi_{3 j}^{2} \\ \phi_{4 j}^{2} & -\phi_{3 j} \phi_{4 j}\end{array}\right)$, we demand that

$$
\frac{\delta \lambda_{j}}{\delta u_{i}}=\frac{1}{2} \operatorname{Tr}\left(\Psi_{j 12} A_{i j}\right), \mathrm{i}=4,5,6
$$

By using of (14) and (15), we present a kind of the integrable couplings of integrable soliton equation hierarchy with self-consistent sources where $n=1,2, \ldots$.

$$
u_{t_{n}}=J \frac{\delta H_{n}}{\delta u}+J \sum_{j=1}^{N} \frac{\delta \lambda_{j}}{\delta u}=J L^{n} \frac{\delta H_{0}}{\delta u}+J \sum_{j=1}^{N} \frac{\delta \lambda_{j}}{\delta u} .
$$

Remark The representation of $\frac{\delta \lambda_{\mathrm{j}}}{\delta u_{i}}$ in [5]-[7] is wrong. In (14) and (15), we correct these errors.

\section{THE INTEGRABLE COUPLINGS OF Li SOLITON Hierarchy With SELF-CONSISTENT Sources}

In [12], Fan presented the zero curvature representation of Li soliton hierarchy. In this section,we consider the following enlarged Li matrix spectral problem

$$
\begin{gathered}
\phi_{x}=U(u, \lambda) \phi \\
U(u, \lambda)=-g_{1}(1)+u_{1}\left(g_{1}(0)+g_{3}(0)\right)+u_{2} g_{2}(0)+u_{3}\left(g_{4}(0)+\right. \\
\left.g_{6}(0)\right)+u_{4} g_{5}(0)
\end{gathered}
$$
i.e.

$$
U(u, \lambda)=\left(\begin{array}{cccc}
-\lambda+u_{1} & u_{1}+u_{2} & u_{3} & u_{3}+u_{4} \\
-u_{1}+u_{2} & \lambda-u_{1} & -u_{3}+u_{4} & -u_{3} \\
0 & 0 & -\lambda+u_{1} & u_{1}+u_{2} \\
0 & 0 & -u_{1}+u_{2} & \lambda-u_{1}
\end{array}\right)
$$

where $\lambda$ is the spectral parameter.

To establish the integrable coupling system of the Lisoliton hierarchy, the stationary zero curvature equation $V x$ $=[U, V]$ of the spectral problem (17) through the generalized Tu scheme [8] is firstly solved. We assume that a solution of $V$ is given by

$$
\begin{gathered}
V=\sum_{m=0}^{\infty} V_{m}(\mathrm{u}) \lambda^{-m}=\sum_{\mathrm{m}=0}^{\infty} \lambda^{-m} \\
\left(\begin{array}{cccc}
a_{m} & b_{m}+c_{m} & d_{m} & e_{m}+f_{m} \\
b_{m}-c_{m} & -a_{m} & e_{m}-f_{m} & -d_{m} \\
0 & 0 & a_{m} & b_{m}+c_{m} \\
0 & 0 & b_{m}-c_{m} & -a_{m}
\end{array}\right)
\end{gathered}
$$

Therefore, the condition (5) gives rise to the following recursion relations:

$$
\left\{\begin{array}{c}
a_{m x}=2 u_{1} b_{m}-2 u_{2} c_{m} \\
b_{m+1}=-u_{2} a_{m}+u_{1} b_{m}-\frac{1}{2} c_{m x} \\
c_{m+1}=-u_{1} a_{m}-\frac{1}{2} b_{m x}+u_{1} c_{m} \\
d_{m x}=2 u_{3} b_{m}-2 u_{4} c_{m}+2 u_{1} e_{m}-2 u_{2} f_{m} \\
e_{m+1}=u_{3} b_{m}-u_{4} a_{m}-u_{2} d_{m}+u_{1} e_{m}-\frac{1}{2} f_{m x} \\
f_{m+1}=u_{3} c_{m}-u_{3} a_{m}-u_{1} d_{m}-\frac{1}{2} e_{m x}+u_{1} f_{m}
\end{array}\right.
$$

If we set $a_{0}=2, b_{0}=c_{0}=d_{0}=e_{0}=f_{0}=0$, we see that all sets of functions $a_{m}, b_{m}, c_{m}, d_{m}, e_{m}, f_{m}$ are uniquely determined. In particular, the first few sets are:

$$
\left(\begin{array}{c}
a_{1}=0, b_{1}=-2 u_{2}, c_{1}=-2 u_{1}, d_{1}=0 \\
e_{1}=-2 u_{4}, f_{1}=-2 u_{3}, a_{2}=u_{1}^{2}-u_{2}^{2}, \\
b_{2}=u_{1 x}-2 u_{1} u_{2}, c_{2}=u_{2 x}-2 u_{1}^{2} \\
d_{2}=2 u_{1} u_{3}-2 u_{3} u_{4} \\
e_{2}=-2 u_{1} u_{4}-2 u_{2} u_{3}+u_{3 x} \\
f_{2}=-4 u_{1} u_{3}+u_{4 x} .
\end{array}\right.
$$

From (19), we can obtain the following recursion relations for $a_{m+1}-c_{m+1}+d_{m+1}-f_{m+1}, b_{m+1}+e_{m+1}, a_{m+1}-$ $c_{m+1}$ and $b_{m+1}$,

$$
\left(\begin{array}{c}
a_{m+1}-c_{m+1}+d_{m+1}-f_{m+1} \\
b_{m+1}+e_{m+1} \\
a_{m+1}-c_{m+1} \\
b_{m+1}
\end{array}\right)=L\left(\begin{array}{c}
a_{m}-c_{m}+d_{m}-f_{m} \\
b_{m}+e_{m} \\
a_{m}-c_{m} \\
b_{m}
\end{array}\right)
$$

where

$$
L=\left(\begin{array}{cccc}
\frac{1}{2} \partial-\partial^{-1} u_{1} \partial & L_{12} & \partial^{-1} u_{3} \partial & L_{14} \\
\frac{1}{2} & 0 & 0 & -u_{3} \\
0 & 0 & \partial^{-1} u_{1} \partial+u_{1} & L_{34} \\
0 & 0 & \frac{1}{2} \partial-u_{2} & 0
\end{array}\right),
$$

where $L_{12}=-\partial^{-1} u_{2} \partial-u_{2}, L_{13}=u_{3}+, L_{14}=\partial^{-1} u_{4} \partial, L_{34}=$ $-\partial^{-1} u_{2} \partial-u_{2}$. 
Considering

$$
\begin{gathered}
V^{(n)}=\sum_{m=0}^{n} V_{m}(u) \lambda^{n-m}+\Delta_{n} \\
\Delta_{n}=\left(\begin{array}{cccc}
-a_{n}+c_{n} & 0 & -d_{n}+f_{n} & 0 \\
0 & a_{n}-c_{n} & 0 & d_{n}-f_{n} \\
0 & 0 & -a_{n}+c_{n} & 0 \\
0 & 0 & 0 & a_{n}-c_{n}
\end{array}\right) .
\end{gathered}
$$
0 ,

from the zero curvature equation $U_{t}-V_{x}^{(n)}+\left[U, V^{(n)}\right]=$

we obtain a new coupling system

$$
U_{t}-\left(\sum_{m=0}^{n} V_{m}(u) \lambda^{n-m}\right)_{x}+\left[U, \sum_{m=0}^{n} V_{m}(u) \lambda^{n-m}\right]-
$$$$
\left[U, \Delta_{n}\right]=0 \text {. }
$$

Substituting (22) into (23), we have

$$
V_{x}^{(n)}-\left[U, V^{(n)}\right]=\left(-a_{n x}+c_{n x}\right) g_{1}+b_{n x} g_{2}+\left(-a_{n x}+c_{n x}\right) g_{3}+
$$$$
\left(-d_{n x}+f_{n x}\right) g_{4}+e_{n x} g_{5}+\left(-d_{n x}+f_{n x}\right) g_{6} \text {. }
$$

Thus the zero curvature equation (23) determines the following system

$$
\begin{aligned}
u_{t}=\left(\begin{array}{l}
u_{1} \\
u_{2} \\
u_{3} \\
u_{4}
\end{array}\right)_{t}=\left(\begin{array}{cccc}
0 & 0 & -\partial & 0 \\
0 & 0 & 0 & \partial \\
-\partial & 0 & \partial & 0 \\
0 & \partial & 0 & -\partial
\end{array}\right)\left(\begin{array}{c}
a_{n}-c_{n}+d_{n}-f_{n} \\
b_{n}+e_{n} \\
a_{n}-c_{n} \\
b_{n}
\end{array}\right) \\
=J\left(\begin{array}{c}
a_{n}-c_{n}+d_{n}-f_{n} \\
b_{n}+e_{n} \\
a_{n}-c_{n} \\
b_{n}
\end{array}\right)=J L^{n}\left(\begin{array}{l}
2 \\
0 \\
2 \\
0
\end{array}\right) .
\end{aligned}
$$

In order to construct non-degenerate bilinear forms, we transform the algebra $s l(4)$ defined by (1) into a vector form.

Define the mapping:

$$
\begin{gathered}
\delta: s l(4) \mapsto R^{6}, \\
\mathrm{~A} \mapsto\left(a_{1}, a_{2}, a_{3}, a_{4}, a_{5}, a_{6}\right)^{T},
\end{gathered}
$$

where $A=a_{1} g_{1}+a_{2} g_{2}+\cdots+a_{6} g_{6} \in \operatorname{sl}(4)$.

This mapping $\delta$ induces a Lie algebra on $\mathrm{R}^{6}$ isomorphic to the matrix Lie algebra sl(4). $\forall a=\left(a_{1}, a_{2}, a_{3}, a_{4}, a_{5}, a_{6}\right)^{\mathrm{T}}, b=$ $\left(b_{1}, b_{2}, b_{3}, b_{4}, b_{5}, b_{6}\right)^{\mathrm{T}} \in R^{6}$, a bilinear form can be defined by

$$
\langle a, b\rangle=a^{T} F b,
$$

where $F$ is a constant matrix. As

$$
(\delta[A, B])^{T}=a^{T} F b=a^{T} R(b),
$$

where

$$
R(b)=\left(\begin{array}{cccccc}
0 & 2 b_{3} & 2 b_{2} & 0 & 2 b_{6} & 2 b_{5} \\
-2 b_{3} & 0 & -2 b_{1} & -2 b_{6} & 0 & -2 b_{4} \\
2 b_{2} & -2 b_{1} & 0 & 2 b_{5} & -2 b_{4} & 0 \\
0 & 0 & 0 & 0 & 2 b_{3} & 2 b_{2} \\
0 & 0 & 0 & -2 b_{3} & 0 & -2 b_{1} \\
0 & 0 & 0 & 2 b_{2} & -2 b_{1} & 0
\end{array}\right) .
$$

According to the symmetry and invariance of the bilinear form, so $F$ and $R(b)$ must satisfy $F^{T}=F, R(b) F=-(R(b) F)^{T}$. So we determine the symmetric matrix $F$ :

$$
F=\left(\begin{array}{cccccc}
1 & 0 & 0 & 1 & 0 & 0 \\
0 & 1 & 0 & 0 & 1 & 0 \\
0 & 0 & -1 & 0 & 0 & -1 \\
1 & 0 & 0 & 0 & 0 & 0 \\
0 & 1 & 0 & 0 & 0 & 0 \\
0 & 0 & -1 & 0 & 0 & 0
\end{array}\right)
$$

We construct the Hamiltonian structure of the Li hierarchy by using of the variational identity [10].

$$
\frac{\delta}{\delta u_{i}}\left\langle V_{n}, \frac{\partial U}{\partial \lambda}\right\rangle=\lambda^{-\gamma} \frac{\partial}{\partial \lambda} \lambda^{\gamma}\left\langle V_{n}, \frac{\partial U}{\partial u_{i}}\right\rangle
$$

Through a direct computation, we obtain

$$
\begin{gathered}
\left\langle V_{n}, \frac{\partial U}{\partial \lambda}\right\rangle=-a_{n}-d_{n},\left\langle V_{n}, \frac{\partial U}{\partial u_{1}}\right\rangle=a_{n}-c_{n}+d_{n}-f_{n}, \\
\left\langle V_{n}, \frac{\partial U}{\partial u_{2}}\right\rangle=b_{n}+e_{n}, \\
\left\langle V_{n}, \frac{\partial U}{\partial u_{3}}\right\rangle=a_{n}-c_{n}, \\
\left\langle V_{n}, \frac{\partial U}{\partial u_{4}}\right\rangle=b_{n .} .
\end{gathered}
$$

Substituting the above formulas into the above identity identity (27), it give s rise to

$$
\frac{\delta}{\delta u}\left\langle V, \frac{\partial U}{\partial \lambda}\right\rangle=\lambda^{-\gamma} \frac{\partial}{\partial \lambda} \lambda^{\gamma}\left(\begin{array}{c}
\left\langle V, \frac{\partial U}{\partial u_{1}}\right\rangle \\
\left\langle V, \frac{\partial U}{\partial u_{2}}\right\rangle \\
\left\langle V, \frac{\partial U}{\partial u_{3}}\right\rangle \\
\left\langle V, \frac{\partial U}{\partial u_{4}}\right\rangle
\end{array}\right),
$$

Comparing the coefficient of $\lambda^{-n-1}$, we get

$$
\begin{gathered}
\left(\frac{\delta}{\delta u_{1}}, \frac{\delta}{\delta u_{2}}, \frac{\delta}{\delta u_{3}}, \frac{\delta}{\delta u_{4}}\right)\left(-a_{n+1}-d_{n+1}\right) \\
=(\gamma-\mathrm{n})\left(\mathrm{a}_{\mathrm{n}}-\mathrm{c}_{\mathrm{n}}+\mathrm{d}_{\mathrm{n}}-\mathrm{f}_{\mathrm{n}}, \mathrm{b}_{\mathrm{n}}+\mathrm{e}_{\mathrm{n}}, \mathrm{a}_{\mathrm{n}}-\mathrm{c}_{\mathrm{n}}, \mathrm{b}_{\mathrm{n}}\right) .
\end{gathered}
$$

To fix the constant $\gamma$, we simplify set $n=1$ in (29) and find that $\gamma=0$. Therefore, we conclude that

$$
G_{n}=\frac{\delta H_{n}}{\delta u}, H_{n}=\int \frac{a_{n+1}+d_{n+1}}{n} \mathrm{~d} x, n \geq 1 .
$$

Hence, the Hamiltonian structure of the coupling $\mathrm{Li}$ hierarchy is constructed as follows

$$
u_{t}=J G_{n}=J \frac{\delta H_{n}}{\delta u} .
$$

Next, we will construct the integrable couplings of $\mathrm{Li}$ equations with self-consistent sources. Considering the auxiliary linear problems

$$
=U\left(u, \lambda_{j}\right)\left(\begin{array}{c}
\phi_{1 j} \\
\phi_{2 j} \\
\phi_{3 j} \\
\phi_{4 j}
\end{array}\right)
$$

$$
\left(\begin{array}{l}
\phi_{1 j} \\
\phi_{2 j} \\
\phi_{3 j} \\
\phi_{4 j}
\end{array}\right)_{t}=V^{(n)}\left(u, \lambda_{j}\right)\left(\begin{array}{l}
\phi_{1 j} \\
\phi_{2 j} \\
\phi_{3 j} \\
\phi_{4 j}
\end{array}\right)
$$

From (14) and (15), we can obtain

$$
\sum_{j=1}^{N} \frac{\delta \lambda_{j}}{\delta u}=\left(\begin{array}{c}
\left\langle\Phi_{1}, \Phi_{2}\right\rangle+\frac{1}{2}\left(\left\langle\Phi_{1}, \Phi_{1}\right\rangle+\left\langle\Phi_{2}, \Phi_{2}\right\rangle\right) \\
\frac{1}{2}\left(\left\langle\Phi_{2}, \Phi_{2}\right\rangle-\left\langle\Phi_{1}, \Phi_{1}\right\rangle\right) \\
\left\langle\Phi_{3}, \Phi_{4}\right\rangle+\frac{1}{2}\left(\left\langle\Phi_{3}, \Phi_{3}\right\rangle+\left\langle\Phi_{4}, \Phi_{4}\right\rangle\right) \\
\frac{1}{2}\left(\left\langle\Phi_{4}, \Phi_{4}\right\rangle-\left\langle\Phi_{3}, \Phi_{3}\right\rangle\right)
\end{array}\right)
$$

where $\Phi_{i}=\left(\phi_{i 1}, \cdots, \phi_{i N}\right)^{T}, \mathrm{i}=1,2,3,4 .\langle\cdot, \cdot\rangle$ denotes the inner product in $\mathrm{R}^{\mathrm{N}}$.

By using the results in $[10,11]$, we have the following equation

$$
\frac{\delta H_{k}}{\delta u}+\sum_{j=1}^{N} \alpha_{j} \frac{\delta \lambda_{j}}{\delta u}=0
$$

where $\alpha_{j}$ are constants, $\frac{\delta H_{k}}{\delta u}$ determines a finite dimensional invariant sets for the flow $u_{t_{n}}=J \frac{\delta H_{n}}{\delta u}, n=1,2, \ldots$.

From (16), we obtain a kind of integrable couplings of $\mathrm{Li}$ soliton equation hierarchy with self-consistent sources as follows

$$
\begin{aligned}
& u_{t}=\left(\begin{array}{l}
u_{1} \\
u_{2} \\
u_{3} \\
u_{4}
\end{array}\right)_{t}=J\left(\begin{array}{c}
a_{n}-c_{n}+d_{n}-f_{n} \\
b_{n}+e_{n} \\
a_{n}-c_{n} \\
b_{n}
\end{array}\right) \\
& -\frac{1}{2} J\left(\begin{array}{c}
-2\left\langle\Phi_{1}, \Phi_{2}\right\rangle-\left\langle\Phi_{1}, \Phi_{1}\right\rangle-\left\langle\Phi_{2}, \Phi_{2}\right\rangle \\
\left\langle\Phi_{1}, \Phi_{1}\right\rangle-\left\langle\Phi_{2}, \Phi_{2}\right\rangle \\
-2\left\langle\Phi_{3}, \Phi_{4}\right\rangle-\left\langle\Phi_{3}, \Phi_{3}\right\rangle-\left\langle\Phi_{4}, \Phi_{4}\right\rangle \\
\left\langle\Phi_{3}, \Phi_{3}\right\rangle-\left\langle\Phi_{4}, \Phi_{4}\right\rangle
\end{array}\right) .
\end{aligned}
$$

A novel integrable coupling system of the Li soliton equation hierarchy with self-consistent sources is presented as follows :

When $n=1$, we have

$$
\begin{aligned}
& u_{1 t}=-2 u_{1 x}-\sum_{j=1}^{N}\left(\left(\phi_{3 j} \phi_{4 j}\right)_{x}+\phi_{3 j} \phi_{3 j x}+\phi_{4 j} \phi_{4 j x}\right), \\
& u_{2 t}=-2 u_{2 x}+\sum_{j=1}^{N}\left(\phi_{4 j} \phi_{4 j x}-\phi_{3 j} \phi_{3 j x}\right), \\
& u_{3 t}=-2 u_{3 x}+\sum_{j=1}^{N}\left(\phi_{3 j} \phi_{3 j x}+\phi_{4 j} \phi_{4 j x}+\left(\phi_{3 j} \phi_{4 j}\right)_{x}\right) \\
&-\sum_{j=1}^{N}\left(\phi_{1 j} \phi_{1 j x}+\phi_{2 j} \phi_{2 j x}+\left(\phi_{1 j} \phi_{2 j}\right)_{x}\right),
\end{aligned}
$$




$$
\begin{aligned}
& u_{4 t}=-2 u_{4 x}+\sum_{j=1}^{N}\left(\phi_{2 j} \phi_{2 j x}+\phi_{3 j} \phi_{3 j x}-\phi_{1 j} \phi_{1 j x}\right. \\
& \left.-\phi_{4 j} \phi_{4 j x}\right) \text {, } \\
& \phi_{1 j x}=\left(-\lambda_{j}+u_{1}\right) \phi_{1 j}+\left(u_{1}+u_{2}\right) \phi_{2 j}+u_{3} \phi_{3 j} \\
& +\left(u_{3}+u_{4}\right) \phi_{4 j} \\
& \phi_{2 j x}=\left(u_{2}-u_{1}\right) \phi_{1 j}+\left(\lambda_{j}-u_{1}\right) \phi_{2 j}+\left(u_{4}-u_{3}\right) \phi_{3 j} \\
& -u_{3} \phi_{4 j} \\
& \phi_{3 j x}=\left(-\lambda_{j}+u_{1}\right) \phi_{3 j}+\left(u_{1}+u_{2}\right) \phi_{4 j} \text {, } \\
& \phi_{4 j x}=\left(u_{2}-u_{1}\right) \phi_{3 j}+\left(\lambda_{j}-u_{1}\right) \phi_{4 j}, j=1, \cdots, N(36) \\
& \text { When } n=2 \text {, we have } \\
& u_{1 t}=u_{2 x x}+2 u_{2} u_{2 x}-6 u_{1} u_{1 x}-\sum_{j=1}^{N}\left(\left(\phi_{3 j} \phi_{4 j}\right)_{x}+\phi_{3 j} \phi_{3 j x}+\phi_{4 j} \phi_{4 j x}\right) \text {, } \\
& u_{2 t}=u_{1 x x}-2\left(u_{1} u_{2}\right)_{x}+\sum_{j=1}^{N}\left(\phi_{4 j} \phi_{4 j x}-\phi_{3 j} \phi_{3 j x}\right) \text {, } \\
& \begin{array}{l}
u_{3 t}=u_{4 x x}-6\left(u_{1} u_{3}\right)_{x}+2\left(u_{2} u_{4}\right)_{x}+ \\
\sum_{j=1}^{N}\left(\phi_{3 j} \phi_{3 j x}+\phi_{4 j} \phi_{4 j x}+\left(\phi_{3 j} \phi_{4 j}\right)_{x}\right)
\end{array} \\
& -\sum_{j=1}^{N}\left(\phi_{1 j} \phi_{1 j x}+\phi_{2 j} \phi_{2 j x}+\left(\phi_{1 j} \phi_{2 j}\right)_{x}\right) \text {, } \\
& u_{4 t}=u_{3 x x}-2\left(u_{1} u_{4}\right)_{x}-2\left(u_{2} u_{3}\right)_{x}+\sum_{j=1}^{N}\left(\phi_{2 j} \phi_{2 j x}+\phi_{3 j} \phi_{3 j x}-\right. \\
& \left.\phi_{1 j} \phi_{1 j x}-\phi_{4 j} \phi_{4 j x}\right) \text {, } \\
& \begin{array}{c}
\phi_{1 j x}=\left(-\lambda_{j}+u_{1}\right) \phi_{1 j}+\left(u_{1}+u_{2}\right) \phi_{2 j}+u_{3} \phi_{3 j} \\
+\left(u_{3}+u_{4}\right) \phi_{4 j}
\end{array} \\
& \phi_{2 j x}=\left(u_{2}-u_{1}\right) \phi_{1 j}+\left(\lambda_{j}-u_{1}\right) \phi_{2 j}+\left(u_{4}-u_{3}\right) \phi_{3 j} \\
& \phi_{3 j x}=\left(-\lambda_{j}+u_{1}\right) \phi_{3 j}+\left(u_{1}+u_{2}\right) \phi_{4 j}, \\
& \phi_{4 j x}=\left(u_{2}-u_{1}\right) \phi_{3 j}+\left(\lambda_{j}-u_{1}\right) \phi_{4 j}, j=1, \cdots, N .
\end{aligned}
$$

We obtain the integrable coupling of $\mathrm{Li}$ equation hierarchy with self-consistent sources. When $u_{3}=u_{4}=0$, (7) can be reduced to the Li equation hierarchy. When $n=2$, $u_{1}=u_{2}=u, u_{3}=u_{4}=0,(37)$ can be reduced to Burgers equation[12] with self-consistent sources

$$
\begin{gathered}
u_{t}=u_{x x}-4 u u_{x x}+\sum_{j=1}^{N}\left(\phi_{2 j} \phi_{2 j x}-\phi_{1 j} \phi_{1 j x}\right),=\left(-\lambda_{j}+u\right) \phi_{1 j}+2 u \phi_{2 j}, \\
\phi_{2 j x}=\left(\lambda_{j}-u\right) \phi_{2 \mathrm{j}}, j=1, \cdots, N .
\end{gathered}
$$

\section{The InTEgRable COUPLINGS OF BKK SOLITON HIERARCHY WITH SELF-CONSISTENT SOURCES}

The BKK hierarchy was brought to prominence by Kupershmidt in [13], where it was attributed to Broer and Kaup. It seems, however, that it should also be attributed to Whitham.. It is frequently just referred to as a "Boussinesq-type" hierarchy. The [14] considers the inverse spectral problem related to the BKK system by some transformation, and the [13] considers the algebraic structure of the BKK system in detail. In this section, we consider the following enlarged BKK matrix spectral problem

$$
\begin{aligned}
& \phi_{x}=U(u, \lambda) \phi \text {, } \\
& U(u, \lambda)=g_{1}(1)+u_{1} g_{1}(0)+\frac{1}{2} u_{2}\left(g_{2}(0)+g_{3}(0)\right)+\frac{1}{2}\left(g_{2}(0)-\right. \\
& \left.g_{3}(0)\right)+u_{3} g_{4}(0)+\frac{1}{2} u_{4}\left(g_{5}(0)+g_{6}(0)\right), \quad(39)
\end{aligned}
$$

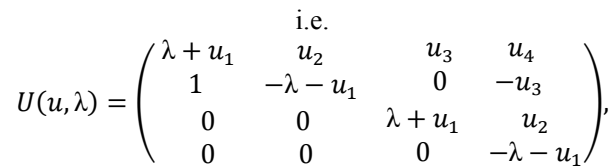

where $\lambda$ is the spectral parameter.

To establish the integrable couplings system of the BKK soliton hierarchy, the stationary zero curvature equation $V x=[U, V]$ of the spectral problem (39) through the generalized Tu scheme [8] is firstly solved. We assume that a solution of $V$ is given by

$$
\begin{gathered}
V=\sum_{m=0}^{\infty} V_{m}(\mathrm{u}) \lambda^{-m}= \\
\sum_{\mathrm{m}=0}^{\infty} \lambda^{-m} \cdot\left(\begin{array}{cccc}
a_{m} & b_{m} & d_{m} & e_{m} \\
c_{m} & -a_{m} & f_{m} & -d_{m} \\
0 & 0 & a_{m} & b_{m} \\
0 & 0 & c_{m} & -a_{m}
\end{array}\right) .
\end{gathered}
$$

Therefore, the condition (5) gives rise to the following recursion relations:

$$
\left\{\begin{array}{c}
a_{m x}=-b_{m}+u_{2} c_{m}, \\
b_{m+1}=u_{2} a_{m}-u_{1} b_{m}+\frac{1}{2} b_{m x}, \\
c_{m+1}=a_{m}-\frac{1}{2} c_{m x}-u_{1} c_{m}, \\
d_{m x}=u_{4} c_{m}-e_{m}+u_{2} f_{m} \\
e_{m+1}=-u_{3} b_{m}+u_{4} a_{m}+u_{2} d_{m}-u_{1} e_{m}+\frac{1}{2} e_{m x}, \\
f_{m+1}=-u_{3} c_{m}+d_{m}-\frac{1}{2} f_{m x}-u_{1} f_{m} .
\end{array}\right.
$$

If we set $a_{0}=1, d_{0}=1, b_{0}=c_{0}=e_{0}=f_{0}=0$, we see that all sets of functions $a_{m}, b_{m}, c_{m}, d_{m}, e_{m}, f_{m}$ are uniquely determined. In particular, the first few sets are:

$$
\left(\begin{array}{c}
a_{1}=0, b_{1}=u_{2}, c_{1}=1, d_{1}=0, e_{1}=u_{2}+u_{4}, \\
f_{1}=1, a_{2}=-\frac{1}{2} u_{2}, b_{2}=\frac{1}{2} u_{2 x}-u_{1} u_{2}, \\
c_{2}=-u_{1}, d_{2}=-\frac{1}{2}\left(u_{2}+u_{4}\right), \\
e_{2}=\frac{1}{2}\left(u_{2}+u_{4}\right)_{x}-u_{1}\left(u_{2}+u_{4}\right)-u_{2} u_{3}, \\
f_{2}=-\left(u_{1}+u_{3}\right) .
\end{array}\right.
$$

From (41), we can obtain the following recursion relations for $2 a_{m+1}+2 d_{m+1}, c_{m+1}+f_{m+1}, 2 a_{m+1}$ and $c_{m+1}$,

$$
\left(\begin{array}{c}
2 a_{m+1}+2 d_{m+1} \\
c_{m+1}+f_{m+1} \\
2 a_{m+1} \\
c_{m+1}
\end{array}\right)=L\left(\begin{array}{c}
2 a_{m}+2 d_{m} \\
c_{m}+f_{m} \\
2 a_{m} \\
c_{m}
\end{array}\right)
$$

wth

$$
L=\left(\begin{array}{cccc}
\frac{1}{2} \partial-\partial^{-1} u_{1} \partial & L_{12} & -\partial^{-1} u_{3} \partial & L_{14} \\
\frac{1}{2} & L_{22} & 0 & -u_{3} \\
0 & 0 & \frac{1}{2} \partial-\partial^{-1} u_{1} \partial & L_{34} \\
0 & 0 & \frac{1}{2} & L_{44}
\end{array}\right) \text {, }
$$

where $L_{12}=-\partial^{-1} u_{2} \partial-u_{2}, L_{22}=-\frac{1}{2} \partial-u_{1}, L_{14}=-\partial^{-1} u_{4} \partial-u_{4}$,

$$
L_{34}=-\partial^{-1} u_{2} \partial-u_{2}, L_{44}=-\frac{1}{2} \partial-u_{1} .
$$

Considering

$$
\begin{gathered}
V^{(n)}=\sum_{m=0}^{n} V_{m}(u) \lambda^{n-m}+\Delta_{n} \\
\Delta_{n}=\left(\begin{array}{cccc}
-c_{n+1} & 0 & -f_{n+1} & 0 \\
0 & c_{n+1} & 0 & f_{n+1} \\
0 & 0 & -c_{n+1} & 0 \\
0 & 0 & 0 & c_{n+1}
\end{array}\right)
\end{gathered}
$$

From the zero curvature equation $U_{t}-V_{x}^{(n)}+\left[U, V^{(n)}\right]=0$, we obtain a new coupling system

$$
\begin{aligned}
& U_{t}-\left(\sum_{m=0}^{n} V_{m}(u) \lambda^{n-m}\right)_{x}+\left[U, \sum_{m=0}^{n} V_{m}(u) \lambda^{n-m}\right]-\frac{\Delta_{n x}}{}+ \\
& {\left[U, \Delta_{n}\right]=0 .}
\end{aligned}
$$

Substituting (44) into (45), we have

$$
\begin{gathered}
V_{x}^{(n)}-\left[U, V^{(n)}\right]=-c_{n+1, x} g_{1}-a_{n+1, x}\left(g_{2}+g_{3}\right)+-f_{n+1, x} g_{4}- \\
d_{n+1, x}\left(g_{5}+g_{6}\right) .
\end{gathered}
$$

Thus the zero curvature equation (45) determines the following system

$$
\begin{aligned}
u_{t}=\left(\begin{array}{l}
u_{1} \\
u_{2} \\
u_{3} \\
u_{4}
\end{array}\right)_{t}=\left(\begin{array}{llcc}
0 & 0 & 0 & \partial \\
0 & 0 & \partial & 0 \\
0 & \partial & 0 & -\partial \\
\partial & \partial & -\partial & 0
\end{array}\right)\left(\begin{array}{c}
-2\left(a_{n+1}+d_{n+1}\right) \\
-\left(c_{n+1}+f_{n+1}\right) \\
-2 a_{n} \\
-c_{n}
\end{array}\right) \\
=\left(\begin{array}{c}
-2\left(a_{n+1}+d_{n+1}\right) \\
-\left(c_{n+1}+f_{n+1}\right) \\
-2 a_{n} \\
-c_{n}
\end{array}\right) J .
\end{aligned}
$$

In order to construct non-degenerate bilinear forms, we transform the algebra $s l(4)$ defined by (1) into a vector form.

Define the mapping: 
$\delta: s l(4) \mapsto R^{6}$

$\mathrm{A} \mapsto\left(a_{1}, a_{2}, a_{3}, a_{4}, a_{5}, a_{6}\right)^{T}$,

where $A=a_{1} g_{1}+\frac{1}{2}\left(a_{2}+a_{3}\right) g_{2}+\frac{1}{2}\left(a_{2}-a_{3}\right) g_{3}+a_{4} g_{4}+$

$\frac{1}{2}\left(a_{5}+a_{6}\right) g_{5}+\frac{1}{2}\left(a_{5}-a_{6}\right) g_{6} \in \operatorname{sl}(4)$.

This mapping $\delta$ induces a Lie algebra on $\mathrm{R}^{6}$ isomorphic to the matrix Lie algebra sl(4). $\forall a=\left(a_{1}, a_{2}, a_{3}, a_{4}, a_{5}, a_{6}\right)^{\mathrm{T}}, b=$ $\left(b_{1}, b_{2}, b_{3}, b_{4}, b_{5}, b_{6}\right)^{\mathrm{T}} \in R^{6}$, a bilinear form can be defined by $\langle a, b\rangle=a^{T} F b$,

where $F$ is a constant matrix. As

$$
(\delta[A, B])^{T}=a^{T} F b=a^{T} R(b),
$$

where

$$
R(b)=\left(\begin{array}{cccccc}
0 & 2 b_{2} & -2 b_{3} & 0 & 2 b_{5} & -2 b_{6} \\
b_{3} & -2 b_{1} & 0 & b_{6} & -2 b_{4} & 0 \\
-b_{2} & 0 & 2 b_{1} & -b_{5} & 0 & 2 b_{4} \\
0 & 0 & 0 & 0 & 2 b_{2} & -2 b_{3} \\
0 & 0 & 0 & b_{3} & -2 b_{1} & 0 \\
0 & 0 & 0 & -b_{2} & 0 & 2 b_{1}
\end{array}\right) .
$$

According to the symmetry and invariance of the bilinear form, so $F$ and $R(b)$ must satisfy $F^{T}=F, R(b) F=-(R(b) F)^{T}$. So we determine the symmetric matrix $F$ :

$$
F=\left(\begin{array}{llllll}
2 & 0 & 0 & 2 & 0 & 0 \\
0 & 0 & 1 & 0 & 0 & 1 \\
0 & 1 & 0 & 0 & 1 & 0 \\
2 & 0 & 0 & 0 & 0 & 0 \\
0 & 0 & 1 & 0 & 0 & 0 \\
0 & 1 & 0 & 0 & 0 & 0
\end{array}\right)
$$

We construct the Hamiltonian structure of the BKK hierarchy by using of the variational identity [10].

$$
\frac{\delta}{\delta u_{i}}\left\langle V_{n}, \frac{\partial U}{\partial \lambda}\right\rangle=\lambda^{-\gamma} \frac{\partial}{\partial \lambda} \lambda^{\gamma}\left\langle V_{n}, \frac{\partial U}{\partial u_{i}}\right\rangle .
$$

Through a direct computation, we obtain

$$
\begin{gathered}
\left\langle V_{n}, \frac{\partial U}{\partial \lambda}\right\rangle=2 a_{n}+2 d_{n}, \\
\left\langle V_{n}, \frac{\partial U}{\partial u_{1}}\right\rangle=2 a_{n}+2 d_{n},\left\langle V_{n}, \frac{\partial U}{\partial u_{2}}\right\rangle=c_{n}+f_{n}, \\
\left\langle V_{n}, \frac{\partial U}{\partial u_{3}}\right\rangle=2 a_{n}, \quad\left\langle V_{n}, \frac{\partial U}{\partial u_{4}}\right\rangle=c_{n} .
\end{gathered}
$$

Substituting the above formulas into the above identity identity (49), it give s rise to

$$
\frac{\delta}{\delta u}\left\langle V, \frac{\partial U}{\partial \lambda}\right\rangle=\lambda^{-\gamma} \frac{\partial}{\partial \lambda} \lambda^{\gamma}\left(\begin{array}{c}
\left\langle V, \frac{\partial U}{\partial u_{1}}\right\rangle \\
\left\langle V, \frac{\partial U}{\partial u_{2}}\right\rangle \\
\left\langle V, \frac{\partial U}{\partial u_{3}}\right\rangle \\
\left\langle V, \frac{\partial U}{\partial u_{4}}\right\rangle
\end{array}\right),
$$

Comparing the coefficient of $\lambda^{-\mathrm{n}-1}$, we get

$$
\begin{aligned}
& \left(\frac{\delta}{\delta u_{1}}, \frac{\delta}{\delta u_{2}}, \frac{\delta}{\delta u_{3}}, \frac{\delta}{\delta u_{4}}\right)\left(2 a_{n+1}+2 d_{n+1}\right) \\
& \quad=(\gamma-n)\left(2 a_{n}+2 d_{n}, c_{n}+f_{n}, 2 a_{n}, c_{n}\right) .
\end{aligned}
$$

To fix the constant $\gamma$, we simplify set $n=1$ in (51) and find that $\gamma=0$. Therefore, we conclude that

$$
G_{n}=\frac{\delta H_{n}}{\delta u}, H_{n}=2 \int \frac{a_{n+2}+d_{n+2}}{n+1} \mathrm{~d} x, n \geq 0 .
$$

Hence, the Hamiltonian structure of the coupling BKK hierarchy is constructed as follows

$$
u_{t}=J G_{n}=J \frac{\delta H_{n}}{\delta u} .
$$

Next, we will construct the integrable couplings of BKK equations with self-consistent sources. Considering the auxiliary linear problems

$$
\begin{aligned}
& \left(\begin{array}{l}
\phi_{1 j} \\
\phi_{2 j} \\
\phi_{3 j} \\
\phi_{4 j}
\end{array}\right)_{x}=U\left(u, \lambda_{j}\right)\left(\begin{array}{l}
\phi_{1 j} \\
\phi_{2 j} \\
\phi_{3 j} \\
\phi_{4 j}
\end{array}\right), \\
& \left(\begin{array}{l}
\phi_{1 j} \\
\phi_{2 j} \\
\phi_{3 j} \\
\phi_{4 j}
\end{array}\right)_{t}=V^{(n)}\left(u, \lambda_{j}\right)\left(\begin{array}{l}
\phi_{1 j} \\
\phi_{2 j} \\
\phi_{3 j} \\
\phi_{4 j}
\end{array}\right) .
\end{aligned}
$$

From (14) and (15), we can obtain

$$
\sum_{j=1}^{N} \frac{\delta \lambda_{j}}{\delta u}=\left(\begin{array}{c}
\left\langle\Phi_{1}, \Phi_{2}\right\rangle \\
\frac{1}{2}\left\langle\Phi_{2}, \Phi_{2}\right\rangle \\
\left\langle\Phi_{3}, \Phi_{4}\right\rangle \\
\frac{1}{2}\left\langle\Phi_{4}, \Phi_{4}\right\rangle
\end{array}\right),
$$

where $\left.\Phi_{i}=\left(\phi_{i 1}, \cdots, \phi_{i N}\right)^{T}, \mathrm{i}=1,2,3,4 .\langle\cdot, \cdot\rangle\right\rangle$ denotes the inner product in $R^{N}$.

By using the results in $[10,11]$, we have the following equation

$$
\frac{\delta H_{k}}{\delta u}+\sum_{j=1}^{N} \alpha_{j} \frac{\delta \lambda_{j}}{\delta u}=0
$$

where $\alpha_{j}$ are constants, $\frac{\delta H_{k}}{\delta u}$ determines a finite dimensional invariant sets for the flow $u_{t_{n}}=J \frac{\delta H_{n}}{\delta u}, n=1,2, \ldots$

From (16), we obtain a kind of integrable couplings of BKK soliton equation hierarchy with self-consistent sources as follows

$$
u_{t}=\left(\begin{array}{l}
u_{1} \\
u_{2} \\
u_{3} \\
u_{4}
\end{array}\right)_{t}=J\left(\begin{array}{c}
2 a_{n}+2 d_{n} \\
c_{n}+f_{n} \\
2 a_{n} \\
c_{n}
\end{array}\right)+\frac{1}{2} J\left(\begin{array}{c}
2\left\langle\Phi_{1}, \Phi_{2}\right\rangle \\
\left\langle\Phi_{2}, \Phi_{2}\right\rangle \\
2\left\langle\Phi_{3}, \Phi_{4}\right\rangle \\
\left\langle\Phi_{4}, \Phi_{4}\right\rangle
\end{array}\right)
$$

A novel integrable coupling system of the BKK soliton equation hierarchy with self-consistent sources is presented as follows :

when $n=1$, we have

$$
\begin{gathered}
u_{1 t}=u_{1 x}+\sum_{j=1}^{N} \phi_{4 j} \phi_{4 j x}, \\
u_{2 t}=u_{2 x}+\sum_{j=1}^{N}\left(\phi_{3 j x} \phi_{4 j}+\phi_{3 j} \phi_{4 j x}\right), \\
u_{3 t}=u_{1 x}+u_{3 x}+\sum_{j=1}^{N}\left(\phi_{2 j} \phi_{2 j x}-\phi_{4 j} \phi_{4 j x}\right) \\
u_{4 t}=u_{2 x}+u_{4 x}+\sum_{j=1}^{N}\left(\phi_{1 j x} \phi_{2 j}+\phi_{1 j} \phi_{2 j x}-\phi_{3 j x} \phi_{4 j}\right. \\
\left.-\phi_{3 j} \phi_{4 j x}\right), \\
\phi_{1 j x}=\left(\lambda_{j}+u_{1}\right) \phi_{1 j}+u_{2} \phi_{2 j}+u_{3} \phi_{3 j}+u_{4} \phi_{4 j}, \\
\phi_{2 j x}=\phi_{1 j}-\left(\lambda_{j}+u_{1}\right) \phi_{2 j}-u_{3} \phi_{4 j}, \\
\phi_{3 j x}=\left(\lambda_{j}+u_{1}\right) \phi_{3 j}+u_{2} \phi_{4 j}, \\
\phi_{4 j x}=\phi_{3 j}-\left(\lambda_{j}+u_{1}\right) \phi_{4 j}, j=1, \cdots, N .
\end{gathered}
$$

when $n=2$, we have

$$
\begin{gathered}
u_{1 t}=-\frac{1}{2} u_{1 x x}-2 u_{1} u_{1 x}+\frac{1}{2} u_{2 x}-\sum_{j=1}^{N} \phi_{4 j} \phi_{4 j x} \\
u_{2 t}=\frac{1}{2} u_{2 x x}-2\left(u_{1} u_{2}\right)_{x}+\sum_{j=1}^{N}\left(\phi_{3 j x} \phi_{4 j}+\phi_{3 j} \phi_{4 j x}\right), \\
u_{3 t}=-\frac{1}{2} u_{3 x x}-2\left(u_{1} u_{3}\right)_{x}-2 u_{1} u_{1 x}-\frac{1}{2} u_{1 x x}+\frac{1}{2} u_{2 x}+\quad \frac{1}{2} u_{4 x} \\
+\sum_{j=1}^{N}\left(\phi_{2 j} \phi_{2 j x}-\phi_{4 j} \phi_{4 j x}\right) \\
u_{4 t}=\frac{1}{2} u_{2 x x}+\frac{1}{2} u_{4 x x}-2\left(u_{1} u_{2}\right)_{x}-2\left(u_{1} u_{4}\right)_{x}-2\left(u_{2} u_{3}\right)_{x}+ \\
\sum_{j=1}^{N}\left(\phi_{1 j x} \phi_{2 j}+\phi_{1 j} \phi_{2 j x}-\phi_{3 j x} \phi_{4 j}-\phi_{3 j} \phi_{4 j x}\right), \\
\phi_{1 j x}=\left(\lambda_{j}+u_{1}\right) \phi_{1 j}+u_{2} \phi_{2 j}+u_{3} \phi_{3 j}+u_{4} \phi_{4 j}, \\
\phi_{2 j x}=\phi_{1 j}-\left(\lambda_{j}+u_{1}\right) \phi_{2 j}-u_{3} \phi_{4 j}, \\
\phi_{3 j x}=\left(\lambda_{j}+u_{1}\right) \phi_{3 j}+u_{2} \phi_{4 j}, \\
\phi_{4 j x}=\phi_{3 j}-\left(\lambda_{j}+u_{1}\right) \phi_{4 j}, j=1, \cdots, N .
\end{gathered}
$$

\section{CONCLUSIONS}

In this paper, we have obtained a new integrable couplings of soliton equations hierarchy with self-consistent sources based on loop algebras $\widetilde{s l}(4)$. In fact, integrable couplings of soliton hierarchy with self-consistent sources in [5-7] were obtained by our method. Our method can be applied to other integrable couplings of soliton hierarchies with self-consistent sources. 


\section{REFERENCES}

[1] V.K. Mel'nikov, "Integration of the Korteweg-de Vries equation with asource,” Inverse Problems, vol.6, 1990, pp: 233-246.

[2] V.K. Mel'nikov, "Integration of the nonlinear Schr odinger equation with a source," Inverse Problems, vol.8, 1992, pp. 133-147.

[3] W.X. Ma,"Soliton,Positon and Negaton Solutions to a Schrödinger Self-consistent sources," J. Phys. Soc. Jpn., vol.72, 2003, pp. 3017-3019.

[4] W.X. Ma,"Complexion solutions of the Korteweg-de Vries equation with self-consistent sources," Chaos, Solitions and Fractals, vol. 26, 2005, pp. 1453-1458.

[5] F.J. Yu and L. Li, "An integrable couplings of Dirac soliton hierarchy with self-consistent sources," Appl. Math. Comput., vol. 207, 2009, pp. 171-177.

[6] F.J. Yu,"A kind of integrable couplings of soliton equations hierarchy with self-consistent sources associated with $\widetilde{s l}(4)$, , Phys. Lett. A, vol. 372, 2008, pp. 6613-6621.

[7] F.J. $\mathrm{Yu}$ and L. Li, "Integrable couplings of C-KdV equations hierarchy with self-consistent sources associated with $\widetilde{s l}(4)$, , Phys. Lett. A, vol. 373, 2009, pp. 1632-1638.
[8] W.X. Ma,"A new hierarchy of Liouville integrable generalized Hamiltonian equations and its reduction," Chin. J. Cont. Math., vol.13, 1992, pp. 115-123.

[9] W.X. Ma and M. Chen, "Hamiltonian and quasi-Hamiltonian structures associated with semi-direct sums of Lie algebras," J. Phys. A: Math. Gen., vol. 39, 2006, pp. 10787-10801.

[10] Y.B. Zeng and Y.S. Li,"The general scheme for higher-order decompositions of zero curvature equations associated with $\widetilde{s l}(2), "$ Acta. Math. Appl., vol.12, 1996, pp. 337-353.

[11] Y.B. Zeng, W.X. Ma and R.L. Lin,"Integration of the soliton hierarchy with self-consistent sources," J. Math. Phys., vol.41, 2000, pp. 5453-5489.

[12] E.G. Fan, "The zero curvature representation for hierarchies of nonlinear evolution equations," Phys. Lett. A, vol. 274, 2000, pp. $135-142$.

[13] B.A. Kupershmidt, "Mathematics of dispersive water waves," Commun. Math. Phys., vol 99, 1985, pp. 51-73.

[14] D.J. Kaup, “A higher-order water wave equation and method for solving it," Prog. Theor. Phys., vol. 54, 1975, pp.396-408. 